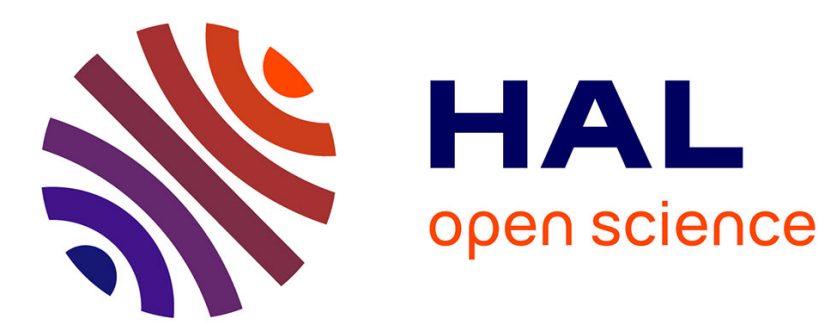

\title{
Insights into the non-mitotic functions of Aurora kinase A: more than just cell division
}

Giulia Bertolin, Marc Tramier

\section{To cite this version:}

Giulia Bertolin, Marc Tramier. Insights into the non-mitotic functions of Aurora kinase A: more than just cell division. Cellular and Molecular Life Sciences, 2020, 77 (6), pp.1031-1047. 10.1007/s00018019-03310-2 . hal-02304767

\section{HAL Id: hal-02304767 \\ https://hal-univ-rennes1.archives-ouvertes.fr/hal-02304767}

Submitted on 12 Feb 2020

HAL is a multi-disciplinary open access archive for the deposit and dissemination of scientific research documents, whether they are published or not. The documents may come from teaching and research institutions in France or abroad, or from public or private research centers.
L'archive ouverte pluridisciplinaire HAL, est destinée au dépôt et à la diffusion de documents scientifiques de niveau recherche, publiés ou non, émanant des établissements d'enseignement et de recherche français ou étrangers, des laboratoires publics ou privés. 
Insights into the non-mitotic functions of Aurora kinase A: more than just cell division

Giulia Bertolin* and Marc Tramier*

Univ Rennes, CNRS, IGDR (Genetics and Development Institute of Rennes), UMR 6290, F-35000 Rennes, France

* Correspondence should be addressed to G.B. (Genetics and Development Institute of Rennes (IGDR), 2, Avenue du Prof. Léon Bernard, 35043 Rennes, France; +33223237516; giulia.bertolin@univrennes1.fr) or to M.T. (Genetics and Development Institute of Rennes (IGDR), 2, Avenue du Prof. Léon Bernard, 35043 Rennes, France; +33223235487; marc.tramier@univ-rennes1.fr) 


\begin{abstract}
AURKA is a serine/threonine kinase overexpressed in several cancers. Originally identified as a protein with multifaceted roles during mitosis, improvements in quantitative microscopy uncovered several nonmitotic roles as well. In physiological conditions, AURKA regulates cilia disassembly, neurite extension, cell motility, DNA replication and senescence programs. In cancer-like contexts, AURKA actively promotes DNA repair, it acts as a transcription factor, promotes cell migration and invasion, and it localises at mitochondria to regulate mitochondrial dynamics and ATP production. Here we review the non-mitotic roles of AURKA, and its partners outside of cell division. In addition, we make an insight on how structural data and quantitative fluorescence microscopy allowed to understand AURKA activation and its interaction with new substrates, highlighting future developments in fluorescence microscopy needed to better understand AURKA functions in vivo. Last, we will recapitulate the most significant AURKA inhibitors currently in clinical trials, and we will explore how the non-mitotic roles of the kinase may provide new insights to ameliorate current pharmacological strategies against AURKA overexpression.
\end{abstract}

Keywords: AURKA, cell cycle, non-mitotic roles, structural data, fluorescence microscopy, FRET. 


\section{Introduction}

From an historical perspective, AURKA was first discovered in 1993 in buddying yeast [1]. The orthologue of AURKA in Saccaromyces cerevisiae, a protein called Ipl1 (Increase-in-ploidy 1), was identified as a mitotic factor controlling chromosome segregation. Since then, two Ipl1 orthologues were found in Xenopus laevis [2-5] and in Drosophila melanogaster [6-9]. In both organisms, the Ipl1 orthologues are kinases with multiple roles at mitosis, including the regulation of centrosomal stability and the faithful construction and maintenance of the mitotic spindle. These roles are shared by the three mammalian orthologues of Ipl1, called Aurora kinases (AURKA, AURKB and AURKC), which were found to be overexpressed in several solid tumours including ovarian, skin, pancreatic and breast cancers [1013]. In particular, the amplification of the AURKA gene, located on chromosome 20p13 [14], is found in a great number of epithelial cancers [13] and in haematological malignancies [15]. The overexpression of AURKA was shown to correlate with highly-proliferative and malignant cancers, with poor outcomes and low survival rates [13,16-18]. Given this large body of evidence and the relevance of this protein in the clinic, extensive efforts were made to understand the molecular functions and partners of the AURKA at cell division.

Today, it is becoming increasingly clear that a multifunctional protein as AURKA has both mitotic and non-mitotic roles. At first, this discovery was made possible by structural data, providing helpful insights on the way the kinase switches between an on and off state. Then, quantitative microscopy approaches as Förster's Resonance Energy Transfer (FRET) paved the way to the monitoring of AURKA activation and activity in live cells. In addition to the discovery of non-mitotic roles, the increasing sensitivity of microscopy tools available nowadays also allowed to uncover new functions of AURKA in different subcellular compartments.

Here, we will make an overview of the non-mitotic roles of AURKA by exploring the roles of the kinas throughout interphase and at different organelles. In addition, we will explore how the discovery of the non-mitotic roles of AURKA largely benefited from cutting-edge technological and methodological capabilities. Last, we will discuss future improvements and strategies in quantitative microscopy potentially allowing to move from in cellulo to in vivo paradigms in the near future. This could be a turning point in ameliorating current pharmacological strategies against AURKA overexpression, which show a poor efficacy in patients [19].

Lessons from biochemistry in detecting AURKA activation and activity: still missing a piece of the puzzle? 
Conventionally, the first approach used to assess the role(s) of a kinase is determining its activity towards a putative substrate. This can be achieved in different ways: among the most common approaches, the activity of a kinase can be tested in vitro. The kinase is purified from bacteria, cells or tissues, and it is used to phosphorylate a putative substrate in presence of ATP $[20,21]$. In this experimental context, the use of radioactive ATP can help discriminating the phosphorylated form of the substrate by the non-phosphorylated one in western-blotting analyses. Otherwise, antibodies raised to recognise the phosphorylation site of a substrate (i.e. phospho-specific antibodies) can be used to track the activity of the kinase towards a putative interactor. This strategy is an alternative to the use of radioactive ATP, although the phosphorylated residue(s) of the substrate must be known, and phosphospecific antibodies are to be manufactured when not commercially available. The purified kinase can also be added directly to cell or tissue lysates, and then processed by biochemical approaches. This allows to explore the capacity of the kinase to phosphorylate a given substrate in its endogenous environment [20]. Biochemical approaches are currently being coupled to quantitative proteomics and mass spectrometry approaches to identify new substrates of the kinase. In this light, finding key substrates of AURKA at different subcellular locations is increasing our knowledge of its multiple roles in the cell, and new roles for AURKA are being discovered thanks to the broadening of its interactome. For instance, the presence of mitochondrial proteins interacting with AURKA led to the discovery of a new function of this kinase in the maintenance of mitochondrial homeostasis [22], while the nuclear proteome of AURKA led to the identification of a novel pathway maintaining cell survival in gastric cancers [23].

When searching for novel roles of a kinase at a given subcellular compartment, it should be taken into consideration that this is often a two-faceted process. Besides the activity towards a substrate, another key parameter to evaluate is the activation of the kinase. Conventionally, the activation results from the autophosphorylation of a Ser, a Thr or a Tyr residue by the kinase itself. In the case of AURKA, its activation results in the autophosphorylation on Thr288 [24-27], which changes the three-dimensional conformation of the catalytic pocket of the kinase. This conformational change was originally detected on the purified protein following crystallography analyses [25]. Activation is currently seen as a "switch-on" signal before AURKA can phosphorylate a target protein and indeed, AURKA autophosphorylation is known to directly increase the catalytic activity of the human and Xenopus kinase [24, 27, 28]. Although there is evidence indicating that a monomer of AURKA can autophosphorylate itself [29], a recent report demonstrated that the autophosphorylation on Thr288 involves a dimer of AURKA, with one monomer of the kinase adding a phosphate group to the other [30]. In both cases, this is considered a priming event, allowing AURKA to interact with the Targeting Protein for XkIp2 (TPX2): thanks to this interaction, crystallography studies showed that TPX2 induces a second conformational change on AURKA [26], 
which then becomes a fully activated kinase capable of interacting with its substrates [26, 31-33]. However, the field is still missing the spatiotemporal resolution of these events: (i) autophosphorylation on Thr288, (ii) interaction with TPX2, (iii) activity towards a substrate. To add a further layer of complexity, whether AURKA activation and activity happen sequentially or simultaneously is still a matter of debate. Structural data showed that autophosphorylated AURKA cannot reach a full activity in the absence of TPX2 [34]. On the contrary, crystallography and Small-angle X-ray scattering (SAXS) data recently showed that the autophosphorylation on Thr288 and the interaction of AURKA with TPX2 are two alternative ways to prime for an active kinase [30], while complementary kinetic measurements showed that these two activation modes can act synergistically [35]. This scenario could potentially reconcile crystallography data with an in vivo report from Toya and colleagues. In the C. elegans embryo, active AURKA was shown to be dephosphorylated on Thr288 when bound to TPX2 on mitotic microtubules [36].

Although structural information greatly improved our understanding of AURKA activation, biochemical and cell biology data were essential to discover how the kinase is dynamically turned off. In cultured cells, AURKA autophosphorylation on Thr288 is targeted by Protein Phosphatase 1 (PP1) [32, 37], which impedes the overall activation of AURKA during interphase. In the interphase-to-mitosis switch, AURKA phosphorylates PP1, and this modification in turn reduces the activity of the phosphatase and it allows AURKA to be fully active during cell division. However, how can AURKA switch from a non-active state to an active one, capable of phosphorylating PP1? Phosphatase Inhibitor 2 (I-2) is the third player of this complex, and it autophosphorylates AURKA on Thr288 at mitosis with an efficacy similar to TPX2, allowing the kinase to activate and to phosphorylate PP1 [38]. The Phosphoprotein Phosphatase 6 (PP6) holoenzyme was also described as another phosphatase for mitotic AURKA [39]. Conversely to PP1, which acts on the free, activated kinase, PP6 was shown to act on AURKA only when in complex with TPX2, substantiating the fact that AURKA deactivation is a timely-regulated process. Recent data also describe Polo-Like Kinase 1 (PLK1), as an additional actor in this intricate scenario [40]. Before mitosis, PP6 maintains AURKA in an "off' state by blocking the access to the Thr288 residue by TPX2. When mitosis progresses, mass spectrometry analyses and in vitro assays demonstrated that PLK1 interacts with and phosphorylates PP6, reducing PP6 activity towards AURKA and thereby allowing for AURKA autophosphorylation. Upon late mitosis, PLK1 abundance decreases and the inhibitory activity of PP6 towards AURKA can start again. At the end of cell division, the activity of PP6 and the one of another phosphatase, Protein Phosphatase 2A (PP2A), fully turns AURKA off and sends the kinase for proteasomal degradation [28]. However, evidence is still fragmentary in this context and it is still not known whether these two phosphatases can act synergistically or in consecutive steps. 
Structural data obtained in the last twenty years lead to the conclusion that AURKA activation and activity should not be considered as static states but rather as dynamic events, potentially intertwined. Although the field benefited from extensive efforts in crystallography and in high-resolution biochemical approaches to gain a more thorough insight into the modes of activation of AURKA, it is also facing a challenge: are we reaching the limit in our understanding of the dynamics of AURKA activation because of the available technical and methodological capabilities? How to reconcile structural data obtained with a purified kinase with its behaviour in a physiological context? Can live imaging techniques take the relay and monitor AURKA activation and activity, providing an insight on the spatiotemporal resolution of these events in living samples?

\section{Tracking AURKA with spatiotemporal resolution}

The attempt of gaining a higher spatiotemporal resolution on the activity of mitotic kinases did not begin with AURKA, but with the closely-related kinase AURKB. AURKB is a kinetochore protein, which warrants a faithful cell division by ensuring the correct attachment of microtubules to kinetochores during mitosis [41]. AURKB shares extensive structural and functional homologies with AURKA [42], and the interplay between these two kinase is a subject of intense investigation. FRET biosensors are fluorescent tools allowing to convert a specific state of a protein into a fluorescent signal [43]. Specifically, FRET biosensors reporting on protein phosphorylation have a sensing unit, capable of switching between a nonphosphorylated and a phosphorylated state. They also have a reporting component, constituted by a donor-acceptor fluorophore pair and which allows to discriminate when the sensing unit is phosphorylated or not. A pioneer study first reported on the benefits of FRET biosensors to understand the regulation of selected pools of AURKB during mitosis [44]. Fuller et al used a small 14 amino acid-long peptide from the AURKB substrate KIF2C and containing the residue targeted by AURKB for phosphorylation (Ser196) [45], a FHA2 domain recognising the phosphorylated residue (Ser196 was replaced by a Thr to facilitate the recognition by the FHA2 domain), and a CFP/YFP donor-acceptor FRET pair. The biosensor was then targeted at selective subcellular locations by adding a centromere- or a histone-specific targeting sequence. As the AURKB biosensor was created after a previous FRET biosensor for PKC [46], it should be noted that phosphorylation is correlated with the absence of FRET in this particular biosensor setup. This first AURKB biosensor was shown to be a valuable tool to follow the spatiotemporal dynamics of AURKB activity. Importantly, it allowed to visualise the phosphorylation of the biosensor at metaphase, and a progressive dephosphorylation during anaphase. Using phospho-specific antibodies, the authors also demonstrated that the endogenous KIF2C undergoes the same dephosphorylation gradient, underlining the pertinence of using a FRET biosensor to detect such modifications in live cells. This 
biosensor paved the way for the use of FRET probes to detect spatiotemporally resolved events during mitosis. A cytosolic version of the AURKB FRET sensor, already created by Fuller et al [44], helped to define a gradient of activity for the mitotic kinase PLK1 (Polo-Like Kinase 1) on the same phosphorylated substrate before and during mitosis [47]. Thanks to FRET microscopy, a peak of activity of PLK1 was detected at pro-metaphase followed by a progressive de-activation until late anaphase.

Given the fact that AURKA and AURKB show a similar consensus phosphorylation motifs [48, 49], this FRET biosensor can be considered as a general reporter of the activity of both AURKA and AURKB, with a specificity towards AURKA or AURKB according to the localisation of the biosensor. In light of this consideration, two recent studies relied on the same probe to unravel the activity of AURKA on microtubules or at kinetochores of Drosophila S2 cells, by using a Tau-specific or a Mis12-specific anchor, respectively $[50,51]$. In these reports, AURKA was shown to phosphorylate kinetochore substrates, which substantiates the identification of AURKA itself at kinetochores [37, 52]. Given the fact that both AURKA and AURKB are found at this compartment, this FRET biosensor should then be used with precaution on kinetochores, as its response to an AURKA- or an AURKB-specific phosphorylation are hardly discriminated. Designing FRET biosensors to probe the activity of AURKA or AURKB towards specific substrates and at given subcellular compartments could be an optimisation strategy to broaden our understanding of the activity of these kinases with a more specific spatiotemporal resolution.

Besides activity, another genetically-encoded biosensor showed that FRET is a powerful approach to unravel kinase activation as well. A full-length AURKA flanked by a GFP/mCherry donoracceptor pair was shown to report on the conformational changes of AURKA upon autophosphorylation on Thr288 [53]. This biosensor not only confirmed structural studies describing that AURKA activation results in conformational changes of the kinetic pocket [25, 30], but also that these changes are propagated to the $\mathrm{N}$ - and $\mathrm{C}$-termini of the kinase. One additional feature of this biosensor is the possibility to replace the endogenous protein with the FRET probe: in cultured cells, the biosensor rescues the spindle defects induced by AURKA knockdown, thereby allowing to measure the activation of the kinase in physiological conditions. As detailed below, this conformational biosensor allowed to discover a new role of AURKA in stabilising microtubule regrowth in $\mathrm{G} 1$. Therefore, new microscopy capabilities were particularly advantageous to uncover a new role of AURKA outside of cell division. This is particularly relevant because the kinase has a very low abundance in this cell cycle phase, which makes it nearly undetectable using bulk biochemical approaches. Given that the biosensor contains the entire AURKA sequence, it also allows to localise the kinase at specific subcellular compartments without adding targeting sequences. This uncovered a pool of the kinase at mitochondria, where it is active and where it regulates mitochondrial dynamics and ATP production [22]. 
An exciting perspective coming up in the near future is the use of kinase FRET biosensors in vivo. This is becoming highly feasible for the substrate-based ERK FRET biosensor (EKAR), which has been employed in C. elegans [54], in zebrafish [55], and in mice [56]. In parallel, conformational FRET biosensors containing the full-length sequence of the kinase could become a promising approach to sense the activation of the kinase. As discussed in the last section of this review, coupling substrate-based biosensors to the AURKA conformational biosensor could lead to the development of multiplex FRET strategies to sense the activation and the activity of AURKA directly in vivo.

\section{Emerging roles of AURKA outside mitosis: novel subcellular locations, unpredicted cell cycle phases.}

The roles and the molecular partners of AURKA at mitosis have extensively been characterised in the context of abnormal cell division and cancer, given the fact that the abundance of AURKA peaks when the cell prepares for cytokinesis. An impressive body of evidence demonstrated that the massive accumulation of AURKA at centrosomes begins during the late $\mathrm{G} 2$ phase and continues throughout the $\mathrm{M}$ phase, where the kinase locates onto the mitotic spindle, to the midzone and the midbody. To help the cell enter and progress throughout cell division, AURKA regulates the stability of the centrosomes, it favours the nucleation of the mitotic spindle from the MTOC and its overall stability, and it coordinates chromosome segregation [13]. Once mitosis is achieved, the mitotic pool of AURKA is dismantled and the protein is degraded at the midbody [57]. To this end, AURKA is targeted by an E3 ubiquitin-protein ligase machinery, the anaphase-promoting complex/cyclosome (APC/C) and its two co-activator subunits $\mathrm{CDC} 20$ and $\mathrm{CDH} 1$ [58], to the proteasome [59]. Although there is general consensus on the degradation of AURKA after mitosis, whether the process is ubiquitin-dependent or -independent is still a matter of debate. On one hand, the E3 ubiquitin-protein ligase CHFR binds to the $\mathrm{N}$-terminal region of AURKA and ubiquitylates the kinase [60], delivering AURKA to the proteasome for degradation. This, together with the dephosphorylation of AURKA Ser51 by the PP2A phosphatase [28, 32, 61], constitutes a hallmark for mitotic exit and it allows the APC/C-CDH1 complex to trigger the degradation of AURKA. On the other hand, work performed in cell culture models showed that the Aurora A kinase interacting protein 1 (AURKAIP1) enhances the binding of Antizyme1 (AZ1) on AURKA [62-64]. AZ1 is an enzyme belonging to the polyamine biosynthesis pathway, and it is known to regulate ubiquitin-independent protein degradation programs [65]. According to its enzymatic function, AZ1 promotes the ubiquitin-independent, but proteasome-dependent degradation of AURKA after mitotic exit [63, 64]. Although the evidence for the importance of these mechanisms in in vivo paradigms remains fragmentary, it is not excluded that both systems co-exist, and potentially with different efficiency rates according to cell types and/or tissues. 
In parallel to its well-described mitotic roles, novel and non-mitotic roles of AURKA are now taking the scene. Their importance is underlined by the fact that AURKA can be activated through uncanonical phosphorylation sites and mechanisms, without the requirement of the full series of molecular interactors present at mitosis $[66,67]$. As detailed below, an interphasic pool of AURKA is present in specialised, non-transformed cells, indicating that the kinase plays physiological roles in specific contexts. In addition, interphase AURKA is overexpressed in various types of cancer stem cells, where it actively participates to cell proliferation acting as a transcription factor. Our broaden knowledge of the non-mitotic roles of AURKA benefited from the availability of more sensitive imaging techniques to monitor them, reinforcing the conclusion that AURKA is a multifunctional kinase intervening throughout the entire cell cycle and at various subcellular locations.

Could the expression of AURKA outside of mitosis be seen as a dysfunctional post-mitotic degradation, potentially leading to a cancer-like situation? The answer to this question has long been debated. On one hand, methodological approaches so far limited our understanding of the non-mitotic functions of AURKA, as the abundance of the protein is nearly undetectable beyond cell division using bulk biochemical approaches. On the other hand, AURKA was shown to have physiological non-mitotic functions in specialised cells - ciliated cells and neurons - at G0/G1, and in specific subcellular compartments - mitochondria - throughout interphase. Are physiological, non-mitotic roles of AURKA present only in specialised cells, or at given organelles? Should tissue specificity be considered when discussing about the non-mitotic roles of AURKA? Are there novel, physiological non-mitotic roles of AURKA yet to be discovered? Recent advances in microscopy capabilities and in high-throughput techniques might help to answer these questions in the future.

\section{Physiological roles of AURKA in non-transformed cells during G0/G1}

The first piece of evidence for a role of AURKA outside of mitosis came from the observation that activated AURKA - autophosphorylated on Thr288 - was found in close proximity of cilia in G0/G1 cells [68]. The primary cilium constitutes a docking platform for many signalling pathways, including the Hedgehog, the WNT, and the Notch pathway (reviewed in [69]). In addition, the primary cilium is a structure sensing extracellular cues in a cell cycle-coordinated fashion: when the cell leaves the quiescent state and re-enters the cell cycle, the primary cilium is dismantled [70]. The autophosphorylation of AURKA in G1 favours the disassembly of cilia in closed co-operation with NEDD9 (HEF1, [71]), a substrate of AURKA acting as a scaffold protein in this process, and the deacetylase HDAC6 [68]. In this signalling cascade, HDAC6 was shown to be directly phosphorylated by AURKA in vitro, and that 
phospho-HDAC6 has an enhanced deacetylase activity [68]. By consequence, phosphorylated HDAC6 induces cilia disassembly more potently than unphosphorylated HDAC6. Since this first discovery, our knowledge of the effectors and substrates of AURKA playing a role in cilia assembly and disassembly greatly increased. The PIFO (Primary Cilia Formation) protein was also shown to interact with AURKA in vitro, and to enhance the autophosphorylation of AURKA during cilia disassembly [72]. Interestingly, whether PIFO acts within the NEDD9-AURKA-HDAC6 pathway or in parallel to it remains to be disclosed. Confirming the importance of AURKA in cilia regulation, several studies reported on independent signalling pathways converging on NEDD9 to activate the NEDD9-AURKA-HDAC6 signalling cascade. NEDD9 was shown to be stabilised by a complex formed by PLK1 and the WNT pathway effector DVL2 (Dishevelled-2) [73]. Once formed, this complex is capable of protecting NEDD9 from degradation, thus allowing AURKA to remain active within the NEDD9-AURKA-HDAC6 pathway and to trigger cilia disassembly. The NEDD9-AURKA-HDAC6 pathway can also be negatively regulated by NPHP2 (Inversin), which can interact in vitro with AURKA and with NEDD9, although to a lower extent [74]. This interaction was proposed to prevent the formation of a functional NEDD9-AURKA-HDAC6 pathway, thereby blocking cilia disassembly through a molecular mechanism yet to be characterised. Last, the $\mathrm{Ca}^{2+}{ }_{-}$ dependent interaction of AURKA with Calmodulin promotes the autophosphorylation of AURKA besides Thr288, i.e. on Ser51, 53 and 54, Ser 66 and 67, and Ser98 [66, 75]. These multiple autophosphorylation events favour the interaction of AURKA with NEDD9, and therefore cilia disassembly. Nevertheless, how this series of autophosphorylation events occurs in space and time is currently unknown. It is tempting to speculate that these modifications also change the three-dimensional conformation of AURKA and in this context, FRET microscopy could help discriminating the autophosphorylation events relative to cilia disassembly from the autophosphorylation on Thr288. This would allow to decipher how these modifications are spatiotemporally resolved and to what extent they have consequences on the activity of AURKA towards cilia-related substrates. Given that AURKA has multiple subcellular locations, the discovery that the release of $\mathrm{Ca}^{2+}$ is mediated directly by the endoplasmic reticulum [66] raises the fascinating hypothesis that this subcellular compartment may regulate the activity of AURKA also beyond the release of second messengers.

Another role played by AURKA during G0/G1 was observed in post-mitotic neurons. These cells are largely quiescent, with an extremely limited capacity to proliferate once differentiated [76]. During neurite extension, autophosphorylated AURKA was shown to be highly expressed and active in cells not actively cycling [77]. In cultured dorsal root ganglia neurons, Mori et al demonstrated the existence of a signalling cascade with AURKA as a key player. The priming event in this case is not the autophosphorylation of AURKA on Thr288, but the phosphorylation of AURKA on Thr287 by PKC (Protein 
Kinase C). This modification favours the binding of TPX2 to AURKA, which in turn is required for the activation of the kinase on Thr288 in this particular cell context. Time-course experiments showed that AURKA undergoes autophosphorylation on Thr288 only after PKC-dependent phosphorylation on Thr287, and this occurs in a TPX2-dependent manner. Once activated, AURKA phosphorylates NDEL1, a central actor for microtubule stability in neurons; the PKC-AURKA-NDEL1 axis was then shown to be mandatory to establish a functional Microtubule Organising Centre (MTOC) during neuronal outgrowth. This report provides a first elegant evidence for a spatiotemporally-resolved interplay between AURKA activation and activity in a non-mitotic context.

After the initial reports on non-mitotic functions of AURKA, the kinase was discovered to play multi-faceted roles during the $\mathrm{G} 1$ phase of the cell cycle. Additionally to its activity in primary cilia and in neurons, it was shown that AURKA is involved in the formation of the pre-replication complex. The prereplication complex ensures the initiation of DNA replication and it activates between late mitosis and early $\mathrm{G1}$ [78]. A key component of this complex is GMNN (Geminin), which is phosphorylated by AURKA during the late phases of mitosis [79, 80]. The AURKA-dependent phosphorylation of GMNN stabilises GMNN itself and it prevents its degradation before the cells enter a new G1 phase. In turn, this event contributes to the proper formation of pre-replication complexes when $\mathrm{G} 1$ begins [79]. Additional evidence for a role of AURKA in G1 was provided by FRET microscopy, where the conformational AURKA FRET biosensor was shown to activate [53]. In this cell cycle phase, centrosomal AURKA controls the stability of the tubulin cytoskeleton re-organising in the cytosol after cell division. AURKA performs this function in cooperation with canonical partners as TPX2 and CEP192, reinforcing the idea that the conformational changes of the kinase rely on similar mechanisms throughout the cell cycle. The physiological non-mitotic roles of AURKA in G0/G1 are recapitulated in Fig. 1.

\section{Cancer-related roles of interphase AURKA}

AURKA participates in DNA repair and in gene transcription

Besides its physiological roles outside of cell division described above, AURKA was reported to have non-mitotic roles in cancer cells as well, illustrated in Fig. 2. One of these novel roles was identified after observing that AURKA regulates DNA damage response paradigms in cellulo and in vivo. Urothelial cell carcinoma samples derived from patients showed that the overexpression of AURKA negatively correlated with the expression of the DNA damage response genes BRCA1 and CHK2 [81]. In ovarian cancer cell models, comparable negative correlations were made for BRCA2 [82]. These data were initially linked to the role of overexpressed AURKA in abrogating the G2 checkpoint induced by DNA 
damage [83]. Then, a recent study pointed at a novel role of AURKA in regulating the activity of poly(ADPribose) 1 (PARP1), a key enzyme for DNA repair. The pharmacological inhibition of AURKA in ovarian carcinoma cells was shown to reduce PARP1 levels, and to promote Non-Homologous End Joining repair (NHEJ) mechanisms [84, 85]. Since NHEJ mechanisms are error-prone, this induces cytotoxicity and cell death: in this light, the induction of NHEJ by the AURKA inhibitor Alisertib was shown to reduce cell growth and survival of ovarian carcinoma cells [84]. This work allowed to revisit the role of AURKA in DNA repair in a non-mitotic perspective. First, PARP-dependent NHEJ paradigms are mostly active in the G1 phase of the cell cycle [85], together with BRCA1 expression starting in $\mathrm{G} 1$ and peaking in the S phase [86, 87]. It should also be kept in mind that PARP1 is a nuclear enzyme and although a direct interaction between AURKA and PARP1 has not been formally demonstrated so far, this work raised the possibility of a mutual regulation between the two proteins at the nucleus.

Such possibility was suggested by the discovery that AURKA is enriched in the nucleus of breast cancer stem cells, and that it enhances their proliferative phenotype [88]. While searching for the molecular mechanisms to explain this phenotype, Zheng et al showed that AURKA localises in the nucleus, where it acts as a transcription factor. Taking advantage of intermolecular FRET, the authors discovered that this novel function of AURKA is favoured by the direct interaction of the kinase with the ribonucleoprotein hnRNP $\mathrm{K}$ in the nucleus. This interaction is mandatory for AURKA to activate MYC transcription, and to enhance breast cancer stem cell proliferation. Interestingly, this function of AURKA in the nucleus does not rely on the kinase activity of AURKA, but it only requires the nuclear localisation of the kinase. The transcriptional activation of MYC by AURKA was also shown to be unrelated with the mitotic roles of the kinase, adding further proof that the spatiotemporal localisation of AURKA outside mitosis greatly influence its oncogenic properties. The relationship between AURKA and cancer stem cells was reinforced by discovering that the suppression of AURKA by pharmacological inhibition or by small interfering RNA blocks the proliferation of colorectal cancer stem cells [89], of epithelial-ovarian cancer stem cells [90], of breast cancer stem cells [88, 91], of glioma stem cells [92], and of leukaemia stem cells [93], where the inhibition of the kinase induces cell death by apoptosis. The majority of cancer stem cells remain in the G0-G1 phase of the cell cycle, in a quiescent state [94]. These cells can re-enter the cell cycle and proliferate if subjected to AURKA silencing or inhibition [89, 90, 92]. However, the molecular mechanisms regulating the roles in cell growth and proliferation played by AURKA in dormant cells remain to be disclosed. In light of the recent report pointing at a role of nuclear AURKA as a transcription enhancer in breast cancer stem cells [88], it would be interesting to monitor if the cancer stem phenotype is induced by nuclear AURKA for different populations of cancer stem cells. 
Unconventional roles of AURKA: a potential link with non-mitotic pools of the kinase?

Nearly one decade ago, AURKA was shown to play uncanonical roles, as in inflammation. First evidence came from epithelial ovarian cancer stem cells, a subpopulation of cancer cells resistant to chemotherapy and capable of re-initiating ovarian cancer. These cells were shown to express both high

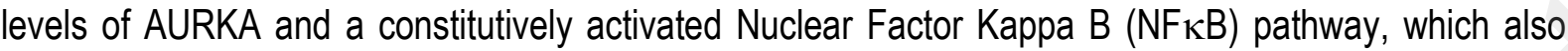
promotes tumour growth and survival [90]. The functional link between the two events came from the evidence that the inhibition of AURKA with MK-5108 induces cell cycle arrest in G2/M, inactivation of the NFKB pathway and cell death [90]. Similarly, in vivo data obtained in paradigms of gastric cancer demonstrated that the inhibition of AURKA blocked the activation of $N F \kappa B$-dependent inflammation paradigms [95]. A concomitant overexpression of AURKA and activation of the NFKB pathway was also reported in Chronic Myeloid Leukaemia, and the inhibition of the kinase or the inactivation of $N F \kappa B$ signalling efficiently triggered cell death [96].

The interaction of AURKA with the MYC pathway was further reinforced by the fact that the overexpression of AURKA often correlates with that of $\mathrm{N}$ - and C-MYC in several types of cancer and cancer cell lines [97-101]. Conversely, the use of RNAi-mediated strategies against C-MYC decreased AURKA-dependent cell proliferation, centrosomal alterations and the transformation potential of cells $[102,103]$. In gastric cancer paradigms, the role of AURKA in enhancing the expression of C-MYC was shown to depend on GSK3B and beta-catenin (CTNNB1), a transcriptional complex which, in turn, activates the transcription of C-MYC [104]. This interplay between AURKA and C-MYC is also a feedback loop, as C-MYC can upregulate the transcription of AURKA in lymphomas [105]. Last, AURKA interacts also with the RAS pathway, enhancing oncogenesis through the interaction with the RAS effector RALA $[106,107]$. Being RALA localised at the plasma membrane and at mitochondria $[107,108]$, its interaction with AURKA heavily supports the involvement of specific pools of the kinase playing different roles in the cancer cell. From a spatial point of view, transcription factors are located in the nucleus and their activation requires a dedicated pool of AURKA. Being $N F_{K} B$ a transcription factor as MYC [88], it is tempting to speculate that the nuclear pool of AURKA is capable of activating several transcription factors at the same time. The same consideration could be made for transcription factors as GSK3B and CTNNB1, and only future data will help elucidating their temporal sequence of activation.

In the attempt to understand the cues triggering senescence in cancer cells, a functional screening using CRISPR/Cas9-based and pharmacological approaches identified AURKA as a key actor in this cellular program [109]. Lung, pancreatic, colorectal, breast, liver and melanoma cancer cells harbouring mutant KRAS and treated with small AURKA inhibitors showed growth rates and morphology 
comparable to senescent cells. An independent study performed in chronic myeloid leukaemia cells also demonstrated that the AKI603 AURKA inhibitor was able to induce cell senescence in cells with wild-type BCL-ABL or its mutated T315I counterpart $[110,111]$. In melanoma, the combination of the AURKA inhibitor Alisertib with an agent activating death receptors - the APO2L/TRAIL ligand - induces a senescence-like state, called therapy-induced senescence (TIS) [112]. TIS cells targeted by Death Receptor 5 antibodies undergo apoptosis, which results in tumour regression in mice xenografts. Although further evidence should be acquired in in vivo paradigms, switching the highly-proliferative cancer cells in cells permanently in the G0/G1 phase represents an intriguing therapeutic perspective to convert cancer in a chronic-like disease. In this light, a better knowledge of the subcellular pool of AURKA responsible for counteracting senescence and its organelle-specific molecular partners will be useful to develop better therapeutic options to induce senescence.

Interphase AURKA is involved in cell migration and invasion

Beyond primary cilia disassembly, the close relationship between AURKA and NEDD9 received further attention by discovering that NEDD9 can be directly phosphorylated by FAK and SRC kinases (reviewed in [113]). In this light, it was reported that the pharmacological inhibition of AURKA delays the migration of ovarian epithelial cancer cells by lowering the abundance of phosphorylated SRC on Tyr416 [114]. Accordingly, an overexpression of AURKA resulted in an increased growth, adhesion and migration of ovarian cancer cells, through the phosphorylation of SRC on the same residue [114]. However, Do et al were not able to fully disclose whether the role of AURKA in cell growth and migration belonged to the mitotic or the non-mitotic roles of AURKA, nor the specific molecular mechanisms involved. To shade light on these two aspects, Mahankali et al discovered that AURKA is the central actor of a non-mitotic cascade involving SRC, FAK and PLD2 (Phospholipase D2) [115]. In interphase cells, SRC is the upstream kinase which can phosphorylate both AURKA on Thr288, and FAK. Once primed by SRC, AURKA can in turn phosphorylate FAK, albeit to a lower efficiency compared to the phosphorylation directly induced by SRC on FAK. Interestingly, PLD2 was shown to enter the play upstream of AURKA by forming a protein-protein complex with the kinase. The lipid mediator phosphatidic acid, which is produced by PLD2, was shown to bind to AURKA and to increase the catalytic activity of the kinase, resulting in an AURKA-dependent stabilisation of tubulin in cultured cells [115]. By positively regulating tubulin integration into microtubules in vitro, the authors proposed that the PLD2-AURKA-SRC-FAK pathway sustains cell migration through microtubule modulation and expansion. A parallel report provided further evidence for an AURKA-related phosphorylation of FAK on Tyr397 in cell models of head and neck squamous cell carcinoma [116]. Abolishing Tyr397 phosphorylation with pharmacological inhibitors of AURKA was shown to lower cell 
migration and invasion in this particular cancer context. However, the exact molecular mechanisms leading to this post-translational modification of FAK and the other players of signalling cascade involving AURKA in this pathological context remain to be elucidated.

In addition to its role on the tubulin stability, AURKA was also shown to actively re-organise the actin cytoskeleton to sustain cell proliferation and migration in breast cancer cell models [117]. When AURKA is overexpressed, it increases the expression levels of SSH1 (Slighshot protein phosphatase 1). SSH1 in turn dephosphorylates CFL (cofilin), a key factor for actin polymerisation [118]. CFL Ser3 dephosphorylation allows the association of CFL with actin and its subsequent integration within actin fibres [117]. Wang and colleagues showed that this molecular pathway falls within the AKT signalling cascade, as the inhibition of PI3K (phosphatidylinositol 3-kinase) suppresses the CFL Ser3 dephosphorylation induced by AURKA through SSH1. They also proved that cell migration was reduced by suppressing this pathway, thereby linking PI3K signalling, AURKA activity and oncogenic progression. Again, it would be interesting to explore whether these events require the prior activation of AURKA on Thr288, what is the molecular mechanisms and/or the cofactors required to activate AURKA (i.e. pure autophosphorylation, TPX2-mediated conformational change or an activator present on actin fibres only), and to what extent AURKA activation and activity are spatiotemporally correlated and lead to the increase of cell motility in this particular context.

AURKA was also shown to play an active role in the Epithelial-Mesenchimal transition (EMT) in oestrogen receptor $\alpha$-positive breast cancer models [119], and in models of pancreatic cancer [120, 121]. The EMT is a transcriptional program with physiological roles from embryogenesis to adulthood; it is also used by cancer cells to promote tumour invasion, metastasis, and drug resistance in the early stages of the tumour development [122]. Breast cancer cells with high invasive capacities show a significant overexpression of AURKA, which is correlated with the acquisition of EMT and of stemness-like properties in vivo [119]. D'assoro et al. showed that these features include the loss of the CD24 cell surface receptor, a mesenchimal morphology, a transcriptomic signature of 11 genes involved in EMT and metastases, together with the overexpression of the SMAD5 and SOX2 markers, which directly promote EMT and stemness. The AURKA inhibitor Alisertib was shown to block these features, arresting the cells at the G1/S checkpoint and inducing autophagy [123], and PARP-dependent apoptosis [119]. However, the molecular mechanism by which AURKA induces EMT remains to be fully disclosed. Two parallel studies identified two direct substrates of AURKA implicated in pancreatic EMT programs, aldehyde dehydrogenase 1 (ALDH1A1) [120] and Twist-related protein 1 (TWIST1) [121]. The AURKA-dependent phosphorylation of both proteins increases their respective enzymatic activity, with a dramatic enhancement of cell motility, drug resistance and the acquisition of a stem-like phenotype. Interestingly, 
these post-translational modifications also create a feedback loop, as the levels of AURKA raise upon the phosphorylation of TWIST1 and of ALDH1A1. However, future studies will explore if these proteins belong to the same molecular pathway, and whether they play a role in EMT paradigms beyond pancreatic cancer.

\section{AURKA regulates mitochondrial homeostasis}

At the cross-road between mitotic and non-mitotic roles of AURKA, several independent studies pointed at a role of the kinase in the maintenance of mitochondrial morphology and dynamics in a kinasedependent manner $[22,108,124]$. AURKA performs these functions due to an unconventional $\mathrm{N}$-terminal Mitochondrial Targeting Sequence (MTS) [22, 124], which allows the targeting and the import of the kinase in the mitochondrial matrix, regardless of the cell cycle phase [22]. At endogenous levels, AURKA ensures mitochondrial fragmentation in interphase cells [22,124] and at mitosis [108]. Before cell division, AURKA is required to localise the small GTPase RALA and its effector RALBP1 at mitochondria, where they facilitate the phosphorylation of DNM1L (Drp1) by the cyclin B-CDK1 complex. DRP1 is one of the main players in mitochondrial fragmentation [125]; the phosphorylation of DRP1 by the AURKA-RALA/RALBP1cyclin B/CDK1 cascade ensures the correct segregation of mitochondria in the daughter cell [108]. However, the interaction of AURKA with DRP1 is not limited to mitosis. At interphase, it has been shown that AURKA directly interacts with DRP1 to ensure organelle fragmentation, and this in a RALAindependent manner [22]. Interestingly, the overexpression of AURKA in cells and in Drosophila induces a marked decrease in the abundance of fission-related proteins, and this shifts the mitochondrial dynamics balance towards elongation. This feature has severe consequences on mitochondrial functionality, as AURKA-driven mitochondrial elongation enhances ATP production from mitochondria [22]. This is consistent with similar data obtained at mitosis, a cell cycle phase where the abundance and the activity of AURKA peak, and where mitochondria show a significant decrease in ATP production if the effector RALA is silenced [108].

Although fascinating, the roles of AURKA at mitochondria raise several questions. Most likely, AURKA has different roles at mitochondria, and thus different partners, at interphase and at mitosis. Could this double-faceted role of AURKA at mitochondria be considered as an intra-mitochondrial moonlighting activity? Is RALA the only gatekeeper helping AURKA to differentiate from interphase-related to mitosisrelated roles? Future data will help elucidating the multiple roles of AURKA at this subcellular compartment. 


\section{Strategies for AURKA inhibition: where do we stand?}

Being a multifunctional kinase with many roles in cancer onset and development, a great effort was made in developing AURKA inhibitors for cancer therapy. AURKA inhibitors are essentially small ATP-analogues targeting the kinetic pocket of the kinase, and they compete against endogenous ATP to abolish the autophosphorylation of Thr288. The consequences of AURKA inhibition are a defective mitotic spindle assembly, leading to a transient, spindle checkpoint-dependent mitotic arrest. However, this mitotic arrest is not maintained, and the cells exit mitosis and begin a new G1 phase. The G1 phase is never completed, as the cells subsequently activate a postmitotic G1 checkpoint, followed by p53dependent or -independent apoptosis programs [126].

Several Aurora kinase inhibitors were developed and released in the market, few of which targeting AURKA with greater specificity, and the majority showing a pan-Aurora inhibition. Very few molecules demonstrated some efficacy in patients during clinical trials, and the evidence of non-mitotic roles of AURKA suggests that further improvements can be made to amplify the efficacy of AURKA inhibitors in killing cancer cells. In this section, we will focus on the available inhibitors specific to AURKA, and we explore possible directions for the development of future drugs.

Alisertib

Alisertib (MLN82374, Millennium pharmaceuticals) is the AURKA inhibitor most extensively studied and tested in clinical trials [127]. It has a 200 -fold better selectivity for AURKA than for AURKB, with an in vitro $\mathrm{IC}_{50}$ of $1.2 \mathrm{nM}$ and negligible off-target effects towards the majority of structurally-related kinases [128]. Interestingly, it was shown to potentiate the effect of specific therapeutic options for Chronic Myeloid Leukaemia as Nilotinib, thanks to the capacity of Alisertib to inhibit BCR-Abl and its T315I mutated form [129]. Alisertib was tested in clinical trials (Phase I/II) for several cancers, including lymphomas, leukaemia, gastric, ovarian and breast cancers [127]. Both alone or in combination with other drugs, Alisertib generally abolishes cell proliferation by inducing a cell cycle arrest, mitotic abnormalities and cell death [130]. However, it also showed a certain degree of toxicity in patients, as neutropenia, nausea and fatigue appear during or after the treatment [127]. Alisertib was the only AURKA inhibitor included in a Phase III trial in 2012. This clinical trial was interrupted in 2015, due to non-significant effects of the molecule on the survival of patients with relapsed or refractory peripheral T-Cell lymphoma. 


\section{VX-689/MK-5108}

This compound, commercialised by Vertex as VX-689 and by Merck as MK-5108, is a potent AURKA inhibitor with a 220-fold better selectivity for AURKA over AURKB, with an $\mathrm{IC}_{50}$ of $0.064 \mathrm{nM}$. VX689/MK-5108 abolishes AURKA autophosphorylation on Thr288 and its activity toward histone H3 [131], and it blocks cell proliferation by arresting cells at the G2/M checkpoint alone or in combination with complementary treatments for Uterine Leiomyosarcoma [131, 132]. VX-680/MK5108, was also shown to be beneficial in in vitro and in mouse models of non-small cell lung cancer with SMARCA4 mutations [133]. AURKA inhibition resulted in the induction of apoptosis and cell death, offering a potential therapeutic perspective for this pathology. A recent Phase I study showed a remarkably low toxicity of this compound in patients with solid tumours [134], making of MK-5108 an interesting candidate for future trials.

\section{ENMD-2076}

ENMD-2076 (EntreMed) has a 25-fold better selectivity for AURKA over AURKB, and an IC 50 of $14 \mathrm{nM}$ [135], although an inhibitory activity towards multiple kinases has been described for this compound [135]. In vitro experiments revealed that ENMD-2076 induces a G2/M checkpoint block and cell death, alone or in combination with radiosensitisation [136]. Phase I trials showed that ENMD-2076 is a lowcytotoxicity compound in patients affected with relapsed or refractory acute myeloid leukaemia, or chronic myelomonocytic leukaemia [137]. To date, several Phase II trials are ongoing.

\section{AURKA and drug resistance}

The combination of AURKA inhibitors with other chemotherapeutic treatments has often been tested as a way to ameliorate the clinical outcome of patients. The association of AURKA inhibitors with microtubule stabilisers (taxanes) showed promising results [138-141]. The AURKA inhibitor MLN8054 (a precursor of Alisertib) or Alisertib itself, in combination with the BRAF inhibitor GSK2118436 and the MEK inhibitor GSK1120212, was shown to have a supplementary anti-proliferative effect in 2D and 3D in cellulo models of melanoma $[142,143]$. However, cell proliferation was not totally abolished and a small fraction of actively proliferating cells were retrieved in the dermal stratum of $3 \mathrm{D}$ cultures, indicating their resistance to drugs. As the kinetic core of AURKA is similar to that of many structurally-related kinases, AURKA inhibitors often show off-target effects in a range of haematological and solid tumours. 
An AURKA-driven resistance program is also activated in in cellulo models of EGFR-mutant nonsmall-cell lung cancer. In this pathological context, the treatment with Osimertinib and Rociletinib - two validated EGFR inhibitors currently used in the clinic - induces drug resistance of unknown origin in nearly $50 \%$ of the patients. Recent evidence correlated the acquisition of resistance programs with the activation of AURKA on Thr288 by TPX2 [144]. The activated kinase phosphorylates the pro-apoptotic factor BIM, preventing apoptosis and leading to cell survival, while the combination of EGFR inhibitors and Alisertib abolishes the AURKA-dependent phosphorylation of BIM and restores apoptosis. The expression of AURKA was also shown to increase in myeloma cells carrying a mutated form of JAK (JAK V617F) [145]. Higher levels of AURKA induced the resistance to cisplatin, a DNA-damaging compound used to induce apoptosis. The cumulative use of cisplatin and of AURKA inhibition-strategies in this context restored apoptosis, reinforcing the role of AURKA in haematological malignancies.

Together, these data indicate the role of AURKA in the acquisition of drug resistance programs. However, it remains to be clarified whether these mechanisms are regulated by a particular subcellular pool of AURKA. In this light, future studies should take this parameter into consideration for a more efficient therapeutic outcome.

\section{Considerations for the design of future AURKA-specific inhibitors}

After two decades in designing and testing AURKA inhibitors, the pertinence of the actual strategy is often questioned. Although these compounds inhibit the activation of AURKA by blocking its autophosphorylation on Thr288 at early mitotic stages, it is becoming evident that some roles of AURKA do not require its kinase activity, and that many new roles of the kinase are being uncovered outside of this cell cycle phase. In this light, it appears that targeting a single pool of AURKA, although abundant and active, is limiting towards the plethora of functions played by this protein and its multiple modes of activation. In addition, AURKA can behave both as an oncogene and as a tumour suppressor, and this in a tissue-specific manner (reviewed in [146]). Therefore, inactivating this kinase can block the development of some tumours in specific tissues where it acts as an oncogene, and trigger the onset of other tumours in cells and tissues where it acts as a tumour suppressor.

A next-generation strategy alternative to the use of ATP-analogues is starting to face in the field: blocking the protein-protein interaction of a kinase with its activator. This strategy has recently been adopted in vitro, where four compounds blocking the interaction between AURKA and TPX2 were isolated [147]. An exciting perspective could be to combine this approach with virtual screenings, taking into account the shape and the electrostatic charge of the kinase [148]. Fluorescence microscopy could also 
help in vivo, by using conformation-sensitive probes capable of showing a FRET-based inhibitory readout in living cells. However, a key parameter to take into consideration is that AURKA can be activated by several proteins and at different organelles. If this reinforces the pertinence of using a combined in silico/in vitro/in cellulo approach to sense the inhibition of specific subcellular pools of the kinase, the activation of AURKA by multiple interactors could add a further layer of complexity to the discovery of pertinent substances for anti-cancer therapies.

As described before, AURKA is not exclusively found at the mitotic spindle but it plays cancerrelevant roles in the nucleus, at mitochondria and on cytoskeletal tracks. In the attempt of optimising current therapies, one central question remains to be answered. Are the actual inhibitors compatible with organelle-specific pools of AURKA? Do they undergo metabolic processing or local inactivation in specific parts of the cell? Future data are required to demonstrate the efficacy of the existing compounds on subcellular pools of the kinase, and organelle-based readouts should be added to the validation of future AURKA inhibitors.

\section{Conclusions and perspectives}

Although considered for nearly two decades a mitotic protein only, the non-mitotic roles of AURKA design not only a widespread distribution of the kinase in different subcellular compartments, but also multifaceted roles for this protein (Fig.1). Significant advances in our understanding of the non-mitotic roles of AURKA came from quantitative microscopy techniques as FRET microscopy [22, 53, 88]. The rise of super-resolution microscopy techniques as Single Molecule Localisation Microscopy (SMLM) [149] could once again revolutionise our understanding of the subcellular locations of AURKA in the near future. However, SMLM techniques currently require long acquisition times, and are largely applicable to fixed samples rather than in the detection of rapid events. A solution could be the use of Structured Illumination Microscopy (SIM) or commercially-available Image Scanning Microscopy approaches [150]. Indeed, Image Scanning Microscopy approaches have already been used to better visualise AURKA at kinetochores [52], but the resolution attained is only around $120 \mathrm{~nm}$ in comparison to $10-20 \mathrm{~nm}$ obtained with SMLM [151]. Taken together, how to benefit from spatiotemporally-resolved activity assays as FRET biosensors and a combined increased resolution in vivo? In this light, FRET by FLINC (Fluorescence Fluctuation Increase by Contact) combined to a super-resolution microscopy technique known as Photochromic Stochastic Optical Fluctuation Imaging (pcSOFI) [152, 153] have already been implemented to assess the activity of the multifunctional kinases PKA and ERK in live cells [154]. It is conceivable that such approaches could be extended to AURKA FRET biosensors, both to detect its 
activation [53] or its activity [44]. That would give a unique insight into the activation or the activity of AURKA in real time and with an increased resolution.

With the expansion of the AURKA interactome [155], it is clear that the creation of novel substratebased FRET biosensors is required. These sensors need to be specific towards (i) a particular AURKA substrate, and (ii) reflect the real activity of the kinase in selected subcellular compartments. This approach would be more pertinent than using a unique substrate-based AURKA biosensor [44] as a general readout for AURKA activity. Not only these biosensors could be used in in vivo models as previously described for ERK, but they could also be used in conjunction with the FRET biosensor reporting on AURKA activation [53]. That would allow to make a correlation between AURKA activation and activity in single cells, with subcellular and temporal resolution. This approach, known as multiplex FRET, has already been implemented for ERK and PKA kinases, using FRET by FLIM [156-158] or fluorescence polarisation microscopy [159] to this end. Interestingly, the work from Ross et al paved the way to the use of three biosensors simultaneously [159]. Constant improvements in the development of fluorescent proteins leads to the fascinating hypothesis that entire signalling pathways could be analysed using these innovative tools. The broad wave of reports illustrating the role of AURKA in primary cilia disassembly clearly indicate that understanding how the kinase interacts with one specific partner (e.g. NEDD9) at a given time and in a given subcellular compartment is a starting point, but not enough to assess the mutual role of each actor within this multi-faceted molecular pathway.

Benefiting from innovative microscopy tools will certainly help us defining signalling pathways where AURKA is involved, but where its role and its partners are still less characterised as in mitochondria or in the nucleus. In addition, the possibility to use these sensors simultaneously in an in vivo context will improve our current strategies for its pharmacological inhibition in epithelial cancer paradigms, which still show poor outcomes in the clinic [19]. 


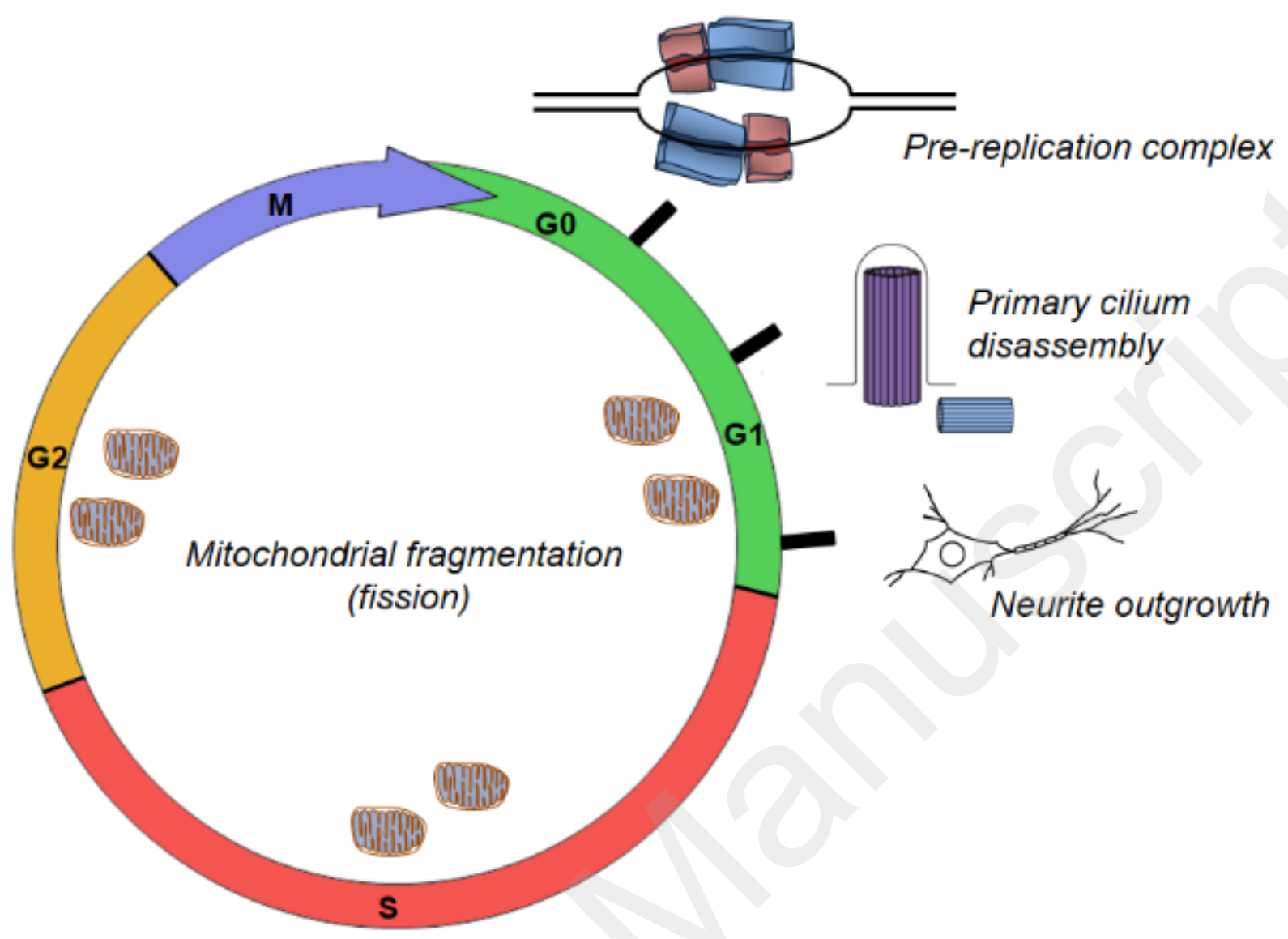

Fig. 1 Schematic overview of the physiological non-mitotic roles of AURKA. To the extent of our knowledge, these roles are confined to G0/G1: the disassembly of primary cilia, neurite outgrowth and the formation of the DNA pre-replication complex. The regulation of mitochondrial dynamics appears to take place throughout the interphase, with AURKA regulating mitochondrial fission when expressed under physiological levels. 


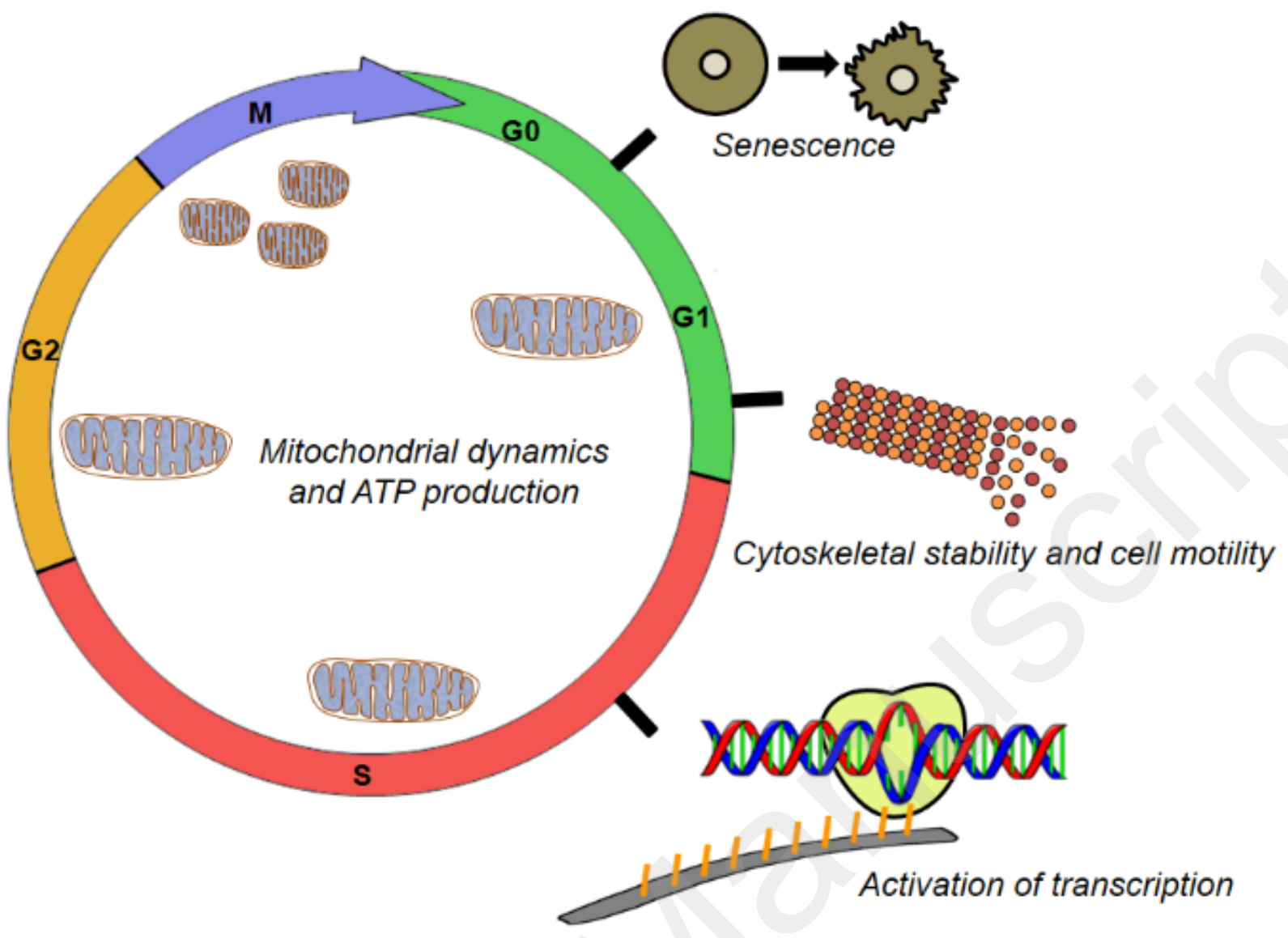

Fig. 2 Schematic overview of the non-mitotic roles of AURKA with relevance for cancer. Cancer-related roles of AURKA outside mitosis are distributed throughout interphase: the activation of senescence programs and cytoskeletal stability are mostly relevant during G0/G1 phase, while in the early $S$ phase we observe the transcriptional activation of key cancer-related factors. Overexpressed AURKA also regulates mitochondrial dynamics, by promoting mitochondrial fusion throughout interphase and fission at mitosis. This has direct consequences on the energetic capabilities of mitochondria, as overexpressed AURKA boosts ATP production. 


\section{Acknowledgements}

We would like to thank C. Prigent, R. Le Borgne, F. Sizaire and EB Gökerküçuk for helpful discussions and feedback concerning AURKA functions and FRET biosensors, and Y. Gautier for help with graphical art. This work was supported by the Centre Nationale de la Recherche Scientifique (CNRS), by the University of Rennes, by the Ligue Contre le Cancer Comité d'Ille et Vilaine et Comité des Côtes d'Armor to G.B. and by the Comité d'Ille et Vilaine, Comité du Maine et Loire et Comité de la Sarthe to MT.

The authors declare that they have no conflicts of interests.

\section{References}

1. Chad CSM, Botsteid D (1993) Isolation and Characterizationof Chromosome-Gain and Increase-inPloidy Mutants in Yeast. Genetics 135:677-691

2. Paris J, Philippe M (1990) Poly(A) metabolism and polysomal recruitment of maternal mRNAs during early Xenopus development. Dev Biol 140:221-224

3. Andrésson T, Ruderman JV (1998) The kinase Eg2 is a component of the Xenopus oocyte progesterone-activated signaling pathway. EMBO $\mathrm{J}$ 17:5627-5637. https://doi.org/10.1093/emboj/17.19.5627

4. Roghi C, Giet R, Uzbekov R, et al (1998) The Xenopus protein kinase pEg2 associates with the centrosome in a cell cycle-dependent manner, binds to the spindle microtubules and is involved in bipolar mitotic spindle assembly. J Cell Sci 111 ( Pt 5):557-572

5. Glover DM, Leibowitz MH, McLean DA, Parry H (1995) Mutations in aurora prevent centrosome separation leading to the formation of monopolar spindles. Cell 81:95-105. https://doi.org/10.1016/0092-8674(95)90374-7

6. Wirtz-Peitz F, Nishimura T, Knoblich JA (2008) Linking cell cycle to asymmetric division: Aurora-A phosphorylates the Par complex to regulate Numb localization. Cell 135:161-173. https://doi.org/10.1016/j.cell.2008.07.049

7. Giet R, McLean D, Descamps S, et al (2002) Drosophila Aurora A kinase is required to localize DTACC to centrosomes and to regulate astral microtubules. J Cell Biol 156:437-451. https://doi.org/10.1083/jcb.200108135

8. Lee C-Y, Andersen RO, Cabernard C, et al (2006) Drosophila Aurora-A kinase inhibits neuroblast self-renewal by regulating aPKC/Numb cortical polarity and spindle orientation. Genes Dev 20:3464-3474. https://doi.org/10.1101/gad.1489406

9. Wang $\mathrm{H}$, Somers $\mathrm{GW}$, Bashirullah A, et al (2006) Aurora-A acts as a tumor suppressor and regulates self-renewal of Drosophila neuroblasts. Genes Dev 20:3453-3463. https://doi.org/10.1101/gad.1487506

10. Meraldi P, Honda R, Nigg EA (2002) Aurora-A overexpression reveals tetraploidization as a major route to centrosome amplification in p53-/- cells. EMBO J 21:483-492 
11. Zhang D, Hirota T, Marumoto T, et al (2004) Cre-loxP-controlled periodic Aurora-A overexpression induces mitotic abnormalities and hyperplasia in mammary glands of mouse models. Oncogene 23:8720-8730. https://doi.org/10.1038/sj.onc.1208153

12. Vader G, Lens SMA (2008) The Aurora kinase family in cell division and cancer. Biochimica et Biophysica Acta (BBA) - Reviews on Cancer 1786:60-72. https://doi.org/10.1016/j.bbcan.2008.07.003

13. Nikonova AS, Astsaturov I, Serebriiskii IG, et al (2013) Aurora A kinase (AURKA) in normal and pathological cell division. Cellular and Molecular Life Sciences 70:661-687. https://doi.org/10.1007/s00018-012-1073-7

14. Isola JJ, Fuqua SAW, Hilsenbeck SG, Waldman FM (1995) Genetic Aberrations Detected by Comparative Genomic Hybridization Predict Outcome in Node-Negative Breast Cancer. 147:7

15. Farag SS (2011) The potential role of Aurora kinase inhibitors in haematological malignancies: Review. British Journal of Haematology 155:561-579. https://doi.org/10.1111/j.13652141.2011.08898.x

16. Zhou H, Kuang J, Zhong L, et al (1998) Tumour amplified kinase STK15/BTAK induces centrosome amplification, aneuploidy and transformation. Nat Genet 20:189-193. https://doi.org/10.1038/2496

17. Miyoshi Y, Iwao K, Egawa C, Noguchi S (2001) Association of centrosomal kinaseSTK15/BTAK mRNA expression with chromosomal instability in human breast cancers. Int J Cancer 92:370-373. https://doi.org/10.1002/ijc.1200

18. Jeng Y-M, Peng S-Y, Lin C-Y, Hsu H-C (2004) Overexpression and Amplification of Aurora-A in Hepatocellular Carcinoma. Clin Cancer Res 10:2065-2071. https://doi.org/10.1158/10780432.CCR-1057-03

19. Bavetsias V, Linardopoulos S (2015) Aurora Kinase Inhibitors: Current Status and Outlook. Front Oncol 5:. https://doi.org/10.3389/fonc.2015.00278

20. Haubrich BA, Swinney DC (2016) Enzyme Activity Assays for Protein Kinases: Strategies to Identify Active Substrates. Curr Drug Discov Technol 13:2-15

21. Knight JDR, Pawson T, Gingras A-C (2013) Profiling the kinome: Current capabilities and future challenges. Journal of Proteomics 81:43-55. https://doi.org/10.1016/j.jprot.2012.10.015

22. Bertolin G, Bulteau A-L, Alves-Guerra M-C, et al (2018) Aurora kinase A localises to mitochondria to control organelle dynamics and energy production. eLife 7:. https://doi.org/10.7554/eLife.38111

23. Kamran M, Long Z-J, Xu D, et al (2017) Aurora kinase A regulates Survivin stability through targeting FBXL7 in gastric cancer drug resistance and prognosis. Oncogenesis 6:e298. https://doi.org/10.1038/oncsis.2016.80

24. Walter AO, Seghezzi W, Korver W, et al (2000) The mitotic serine/threonine kinase Aurora2/AIK is regulated by phosphorylation and degradation. Oncogene 19:4906-4916. https://doi.org/10.1038/sj.onc.1203847

25. Cheetham GMT (2002) Crystal Structure of Aurora-2, an Oncogenic Serine/Threonine Kinase. Journal of Biological Chemistry 277:42419-42422. https://doi.org/10.1074/jbc.C200426200 
26. Bayliss R, Sardon T, Vernos I, Conti E (2003) Structural basis of Aurora-A activation by TPX2 at the mitotic spindle. Molecular cell 12:851-862

27. Zhang $\mathrm{Y}, \mathrm{Ni}$ J, Huang $\mathrm{Q}$, et al (2007) Identification of the auto-inhibitory domains of Aurora-A kinase. Biochemical and Biophysical Research Communications 357:347-352. https://doi.org/10.1016/j.bbrc.2007.03.129

28. Littlepage LE, Wu H, Andresson T, et al (2002) Identification of phosphorylated residues that affect the activity of the mitotic kinase Aurora-A. Proc Natl Acad Sci USA 99:15440-15445. https://doi.org/10.1073/pnas.202606599

29. Dodson CA, Yeoh S, Haq T, Bayliss R (2013) A Kinetic Test Characterizes Kinase Intramolecular and Intermolecular Autophosphorylation Mechanisms. Sci Signal 6:ra54-ra54. https://doi.org/10.1126/scisignal.2003910

30. Zorba A, Buosi V, Kutter S, et al (2014) Molecular mechanism of Aurora A kinase autophosphorylation and its allosteric activation by TPX2. Elife 3:e02667

31. Kufer TA, Silljé HHW, Körner R, et al (2002) Human TPX2 is required for targeting Aurora-A kinase to the spindle. J Cell Biol 158:617-623. https://doi.org/10.1083/jcb.200204155

32. Eyers PA, Erikson E, Chen LG, Maller JL (2003) A novel mechanism for activation of the protein kinase Aurora A. Current Biology 13:691-697

33. Brunet S, Sardon T, Zimmerman T, et al (2004) Characterization of the TPX2 Domains Involved in Microtubule Nucleation and Spindle Assembly in Xenopus Egg Extracts. Mol Biol Cell 15:53185328. https://doi.org/10.1091/mbc.E04-05-0385

34. Burgess SG, Bayliss R (2015) The structure of C290A:C393A Aurora A provides structural insights into kinase regulation. Acta Crystallogr $F$ Struct Biol Commun 71:315-319. https://doi.org/10.1107/S2053230X15002290

35. Dodson CA, Bayliss R (2012) Activation of Aurora-A kinase by protein partner binding and phosphorylation are independent and synergistic. J Biol Chem 287:1150-1157. https://doi.org/10.1074/jbc.M111.312090

36. Toya M, Terasawa M, Nagata K, et al (2011) A kinase-independent role for Aurora A in the assembly of mitotic spindle microtubules in Caenorhabditis elegans embryos. Nature Cell Biology 13:708714. https://doi.org/10.1038/ncb2242

37. Katayama H, Sasai K, Kloc M, et al (2008) Aurora kinase-A regulates kinetochore/chromatin associated microtubule assembly in human cells. Cell Cycle 7:2691-2704. https://doi.org/10.4161/cc.7.17.6460

38. Satinover DL, Leach CA, Stukenberg PT, Brautigan DL (2004) Activation of Aurora-A kinase by protein phosphatase inhibitor-2, a bifunctional signaling protein. PNAS 101:8625-8630. https://doi.org/10.1073/pnas.0402966101

39. Zeng K, Bastos RN, Barr FA, Gruneberg U (2010) Protein phosphatase 6 regulates mitotic spindle formation by controlling the T-loop phosphorylation state of Aurora A bound to its activator TPX2. J Cell Biol 191:1315-1332. https://doi.org/10.1083/jcb.201008106 
40. Kettenbach AN, Schlosser KA, Lyons SP, et al (2018) Global assessment of its network dynamics reveals that the kinase Plk1 inhibits the phosphatase PP6 to promote Aurora A activity. Sci Signal 11:. https://doi.org/10.1126/scisignal.aaq1441

41. Krenn V, Musacchio A (2015) The Aurora B Kinase in Chromosome Bi-Orientation and Spindle Checkpoint Signaling. Front Oncol 5:. https://doi.org/10.3389/fonc.2015.00225

42. Willems E, Dedobbeleer M, Digregorio M, et al (2018) The functional diversity of Aurora kinases: a comprehensive review. Cell Div 13:. https://doi.org/10.1186/s13008-018-0040-6

43. Greenwald EC, Mehta S, Zhang J (2018) Genetically Encoded Fluorescent Biosensors Illuminate the Spatiotemporal Regulation of Signaling Networks. Chem Rev 118:11707-11794. https://doi.org/10.1021/acs.chemrev.8b00333

44. Fuller BG, Lampson MA, Foley EA, et al (2008) Midzone activation of aurora B in anaphase produces an intracellular phosphorylation gradient. Nature 453:1132-1136. https://doi.org/10.1038/nature06923

45. Andrews PD, Ovechkina $Y$, Morrice N, et al (2004) Aurora B Regulates MCAK at the Mitotic Centromere. Developmental Cell 6:253-268. https://doi.org/10.1016/S1534-5807(04)00025-5

46. Violin JD, Zhang J, Tsien RY, Newton AC (2003) A genetically encoded fluorescent reporter reveals oscillatory phosphorylation by protein kinase C. The Journal of Cell Biology 161:899-909. https://doi.org/10.1083/jcb.200302125

47. Macůrek L, Lindqvist $A$, Lim D, et al (2008) Polo-like kinase-1 is activated by aurora $A$ to promote checkpoint recovery. Nature 455:119-123. https://doi.org/10.1038/nature07185

48. Cheeseman IM, Anderson S, Jwa M, et al (2002) Phospho-Regulation of Kinetochore-Microtubule Attachments by the Aurora Kinase Ipl1p. Cell 111:163-172. https://doi.org/10.1016/S00928674(02)00973-X

49. Lens SMA, Voest EE, Medema RH (2010) Shared and separate functions of polo-like kinases and aurora kinases in cancer. Nature Reviews Cancer 10:825-841. https://doi.org/10.1038/nrc2964

50. Ye AA, Deretic J, Hoel CM, et al (2015) Aurora A Kinase Contributes to a Pole-Based Error Correction Pathway. Curr Biol 25:1842-1851. https://doi.org/10.1016/j.cub.2015.06.021

51. Ye AA, Torabi J, Maresca TJ (2016) Aurora A Kinase Amplifies a Midzone Phosphorylation Gradient to Promote High-Fidelity Cytokinesis. Biol Bull 231:61-72. https://doi.org/10.1086/689591

52. Courtheoux T, Diallo A, Damodaran AP, et al (2018) Aurora A kinase activity is required to maintain an active spindle assembly checkpoint during prometaphase. Journal of Cell Science 131:jcs191353. https://doi.org/10.1242/jcs.191353

53. Bertolin G, Sizaire F, Herbomel G, et al (2016) A FRET biosensor reveals spatiotemporal activation and functions of aurora kinase $A$ in living cells. Nature Communications 7:12674. https://doi.org/10.1038/ncomms12674

54. de la Cova C, Townley R, Regot S, Greenwald I (2017) A Real-Time Biosensor for ERK Activity Reveals Signaling Dynamics during C. elegans Cell Fate Specification. Developmental Cell 42:542553.e4. https://doi.org/10.1016/j.devcel.2017.07.014 
55. Mayr V, Sturtzel C, Stadler M, et al (2018) Fast Dynamic in vivo Monitoring of Erk Activity at Single Cell Resolution in DREKA Zebrafish. Front Cell Dev Biol 6:. https://doi.org/10.3389/fcell.2018.00111

56. Konishi Y, Terai K, Furuta Y, et al (2018) Live-Cell FRET Imaging Reveals a Role of Extracellular Signal-Regulated Kinase Activity Dynamics in Thymocyte Motility. iScience 10:98-113. https://doi.org/10.1016/j.isci.2018.11.025

57. Floyd S, Pines J, Lindon C (2008) APC/CCdh1 Targets Aurora Kinase to Control Reorganization of the Mitotic Spindle at Anaphase. Current Biology 18:1649-1658. https://doi.org/10.1016/j.cub.2008.09.058

58. Alfieri C, Zhang S, Barford D (2017) Visualizing the complex functions and mechanisms of the anaphase promoting complex/cyclosome (APC/C). Open Biol 7:170204. https://doi.org/10.1098/rsob.170204

59. Castro A, Arlot-Bonnemains Y, Vigneron S, et al (2002) APC/Fizzy-Related targets Aurora-A kinase for proteolysis. EMBO reports 3:457-462. https://doi.org/10.1093/embo-reports/kvf095

60. Yu X, Minter-Dykhouse K, Malureanu L, et al (2005) Chfr is required for tumor suppression and Aurora A regulation. Nat Genet 37:401-406. https://doi.org/10.1038/ng1538

61. Horn SR, Thomenius MJ, Johnson ES, et al (2011) Regulation of mitochondrial morphology by APC/CCdh1-mediated control of Drp1 stability. Molecular biology of the cell 22:1207-1216

62. Kiat LS, Hui KM, Gopalan G (2002) Aurora-A Kinase Interacting Protein (AIP), a Novel Negative Regulator of Human Aurora-A Kinase. J Biol Chem 277:45558-45565. https://doi.org/10.1074/jbc.M206820200

63. Lim SK, Gopalan G (2007) Antizyme1 mediates AURKAIP1-dependent degradation of Aurora-A. Oncogene 26:6593-6603. https://doi.org/10.1038/sj.onc.1210482

64. Lim SK, Gopalan G (2007) Aurora-A kinase interacting protein 1 (AURKAIP1) promotes Aurora-A degradation through an alternative ubiquitin-independent pathway. Biochem J 403:119-127. https://doi.org/10.1042/BJ20061272

65. Qiu S, Liu J, Xing F (2017) Antizyme inhibitor 1: a potential carcinogenic molecule. Cancer Sci 108:163-169. https://doi.org/10.1111/cas.13122

66. Plotnikova OV, Pugacheva EN, Dunbrack RL, Golemis EA (2010) Rapid calcium-dependent activation of Aurora-A kinase. Nat Commun 1:1-8. https://doi.org/10.1038/ncomms1061

67. Reboutier D, Troadec M-B, Cremet J-Y, et al (2012) Nucleophosmin/B23 activates Aurora A at the centrosome through phosphorylation of serine 89. The Journal of Cell Biology 197:19-26. https://doi.org/10.1083/jcb.201107134

68. Pugacheva EN, Jablonski SA, Hartman TR, et al (2007) HEF1-Dependent Aurora A Activation Induces Disassembly of the Primary Cilium. Cell 129:1351-1363. https://doi.org/10.1016/j.cell.2007.04.035

69. Wheway G, Nazlamova L, Hancock JT (2018) Signaling through the Primary Cilium. Front Cell Dev Biol 6:. https://doi.org/10.3389/fcell.2018.00008 
70. Pan J, Seeger-Nukpezah T, Golemis EA (2013) The role of the cilium in normal and abnormal cell cycles: emphasis on renal cystic pathologies. Cell Mol Life Sci 70:1849-1874. https://doi.org/10.1007/s00018-012-1052-z

71. Pugacheva EN, Golemis EA (2005) The focal adhesion scaffolding protein HEF1 regulates activation of the Aurora-A and Nek2 kinases at the centrosome. Nature Cell Biology 7:937-946. https://doi.org/10.1038/ncb1309

72. Kinzel D, Boldt K, Davis EE, et al (2010) Pitchfork Regulates Primary Cilia Disassembly and LeftRight Asymmetry. Developmental Cell 19:66-77. https://doi.org/10.1016/j.devcel.2010.06.005

73. Lee KH, Johmura Y, Yu L-R, et al (2012) Identification of a novel Wnt5a-CK1ع-Dvl2-PIk1-mediated primary cilia disassembly pathway: Primary cilia disassembly by the Dvl2-PIk1 complex. The EMBO Journal 31:3104-3117. https://doi.org/10.1038/emboj.2012.144

74. Mergen M, Engel C, Muller B, et al (2013) The nephronophthisis gene product NPHP2/Inversin interacts with Aurora A and interferes with HDAC6-mediated cilia disassembly. Nephrology Dialysis Transplantation 28:2744-2753. https://doi.org/10.1093/ndt/gft316

75. Plotnikova OV, Nikonova AS, Loskutov YV, et al (2012) Calmodulin activation of Aurora-A kinase (AURKA) is required during ciliary disassembly and in mitosis. Molecular Biology of the Cell 23:2658-2670. https://doi.org/10.1091/mbc.e11-12-1056

76. Frade JM, Ovejero-Benito MC (2015) Neuronal cell cycle: the neuron itself and its circumstances. Cell Cycle 14:712-720. https://doi.org/10.1080/15384101.2015.1004937

77. Mori D, Yamada M, Mimori-Kiyosue Y, et al (2009) An essential role of the aPKC-Aurora A-NDEL1 pathway in neurite elongation by modulation of microtubule dynamics. Nature Cell Biology 11:10571068. https://doi.org/10.1038/ncb1919

78. Bleichert F, Botchan MR, Berger JM (2017) Mechanisms for initiating cellular DNA replication. Science 355:eaah6317. https://doi.org/10.1126/science.aah6317

79. Tsunematsu T, Takihara Y, Ishimaru N, et al (2013) Aurora-A controls pre-replicative complex assembly and DNA replication by stabilizing geminin in mitosis. Nature Communications 4:1885. https://doi.org/10.1038/ncomms2859

80. Tsunematsu T, Arakaki R, Yamada A, et al (2015) The Non-Canonical Role of Aurora-A in DNA Replication. Front Oncol 5:. https://doi.org/10.3389/fonc.2015.00187

81. Veerakumarasivam A, Goldstein LD, Saeb-Parsy K, et al (2008) AURKA overexpression accompanies dysregulation of DNA-damage response genes in invasive urothelial cell carcinoma. Cell Cycle 7:3525-3533. https://doi.org/10.4161/cc.7.22.7042

82. Yang G, Chang B, Yang F, et al (2010) Aurora Kinase A Promotes Ovarian Tumorigenesis through Dysregulation of the Cell Cycle and Suppression of BRCA2. Clin Cancer Res 16:3171-3181. https://doi.org/10.1158/1078-0432.CCR-09-3171

83. Marumoto T, Hirota T, Morisaki T, et al (2002) Roles of aurora-A kinase in mitotic entry and G2 checkpoint in mammalian cells. Genes to Cells 7:1173-1182. https://doi.org/10.1046/j.13652443.2002.00592.x 
84. Do T-V, Hirst J, Hyter S, et al (2017) Aurora A kinase regulates non-homologous end-joining and poly(ADP-ribose) polymerase function in ovarian carcinoma cells. Oncotarget 8:50376-50392. https://doi.org/10.18632/oncotarget.18970

85. Ray Chaudhuri A, Nussenzweig A (2017) The multifaceted roles of PARP1 in DNA repair and chromatin remodelling. Nat Rev Mol Cell Biol 18:610-621. https://doi.org/10.1038/nrm.2017.53

86. Vaughn JP, Davis PL, Jarboe MD, et al (1996) BRCA1 expression is induced before DNA synthesis in both normal and tumor-derived breast cells. Cell Growth \& Differentiation 7:711

87. Ruffner H, Verma IM (1997) BRCA1 is a cell cycle-regulated nuclear phosphoprotein. Proc Natl Acad Sci U S A 94:7138-7143

88. Zheng F, Yue C, Li G, et al (2016) Nuclear AURKA acquires kinase-independent transactivating function to enhance breast cancer stem cell phenotype. Nature Communications 7:10180. https://doi.org/10.1038/ncomms10180

89. Cammareri P, Scopelliti A, Todaro M, et al (2010) Aurora-A Is Essential for the Tumorigenic Capacity and Chemoresistance of Colorectal Cancer Stem Cells. Cancer Res 70:4655-4665. https://doi.org/10.1158/0008-5472.CAN-09-3953

90. Chefetz I, Holmberg JC, Alvero AB, et al (2011) Inhibition of Aurora-A kinase induces cell cycle arrest in epithelial ovarian cancer stem cells by affecting NFKB pathway. Cell Cycle 10:2206-2214. https://doi.org/10.4161/cc.10.13.16348

91. Zheng F-M, Long Z-J, Hou Z-J, et al (2014) A novel small molecule aurora kinase inhibitor attenuates breast tumor-initiating cells and overcomes drug resistance. Mol Cancer Ther 13:19912003. https://doi.org/10.1158/1535-7163.MCT-13-1029

92. Mannino M, Gomez-Roman N, Hochegger H, Chalmers AJ (2014) Differential sensitivity of Glioma stem cells to Aurora kinase A inhibitors: implications for stem cell mitosis and centrosome dynamics. Stem Cell Res 13:135-143. https://doi.org/10.1016/j.scr.2014.05.001

93. Yang J, Ikezoe T, Nishioka C, et al (2013) CD34+/CD38- acute myelogenous leukemia cells aberrantly express Aurora kinase A. Int J Cancer 133:2706-2719. https://doi.org/10.1002/ijc.28277

94. Clevers $H$ (2011) The cancer stem cell: premises, promises and challenges. Nature Medicine 17:313-319. https://doi.org/10.1038/nm.2304

95. Katsha A, Soutto M, Sehdev V, et al (2013) Aurora Kinase A Promotes Inflammation and Tumorigenesis in Mice and Human Gastric Neoplasia. Gastroenterology 145:. https://doi.org/10.1053/j.gastro.2013.08.050

96. Wei T-YW, Wu P-Y, Wu T-J, et al (2017) Aurora A and NF-kB Survival Pathway Drive Chemoresistance in Acute Myeloid Leukemia via the TRAF-Interacting Protein TIFA. Cancer Res 77:494-508. https://doi.org/10.1158/0008-5472.CAN-16-1004

97. Neben K, Korshunov A, Benner A, et al (2004) Microarray-based screening for molecular markers in medulloblastoma revealed STK15 as independent predictor for survival. Cancer Res 64:31033111 
98. Dimova I, Raitcheva S, Dimitrov R, et al (2006) Correlations between c-myc gene copy-number and clinicopathological parameters of ovarian tumours. European Journal of Cancer 42:674-679. https://doi.org/10.1016/j.ejca.2005.11.022

99. Lassmann S, Shen Y, Jütting U, et al (2007) Predictive value of Aurora-A/STK15 expression for late stage epithelial ovarian cancer patients treated by adjuvant chemotherapy. Clin Cancer Res 13:4083-4091. https://doi.org/10.1158/1078-0432.CCR-06-2775

100. Otto T, Horn S, Brockmann M, et al (2009) Stabilization of N-Myc is a critical function of Aurora A in human neuroblastoma. Cancer Cell 15:67-78. https://doi.org/10.1016/j.ccr.2008.12.005

101. Beltran H, Rickman DS, Park K, et al (2011) Molecular characterization of neuroendocrine prostate cancer and identification of new drug targets. Cancer Discov 1:487-495. https://doi.org/10.1158/2159-8290.CD-11-0130

102. Yang H, Ou CC, Feldman RI, et al (2004) Aurora-A kinase regulates telomerase activity through cMyc in human ovarian and breast epithelial cells. Cancer Res 64:463-467

103. Yang S, He S, Zhou X, et al (2010) Suppression of Aurora-A oncogenic potential by c-Myc downregulation. Exp Mol Med 42:759-767. https://doi.org/10.3858/emm.2010.42.11.077

104. Dar AA, Belkhiri A, El-Rifai W (2009) The aurora kinase A regulates GSK-3beta in gastric cancer cells. Oncogene 28:866-875. https://doi.org/10.1038/onc.2008.434

105. den Hollander J, Rimpi S, Doherty JR, et al (2010) Aurora kinases A and B are up-regulated by Myc and are essential for maintenance of the malignant state. Blood 116:1498-1505. https://doi.org/10.1182/blood-2009-11-251074

106. Wu J-C, Chen T-Y, Yu C-TR, et al (2005) Identification of V23RalA-Ser194 as a Critical Mediator for Aurora-A-induced Cellular Motility and Transformation by Small Pool Expression Screening. J Biol Chem 280:9013-9022. https://doi.org/10.1074/jbc.M411068200

107. Lim K-H, Brady DC, Kashatus DF, et al (2010) Aurora-A Phosphorylates, Activates, and Relocalizes the Small GTPase RalA. Molecular and Cellular Biology 30:508-523. https://doi.org/10.1128/MCB.00916-08

108. Kashatus DF, Lim K-H, Brady DC, et al (2011) RALA and RALBP1 regulate mitochondrial fission at mitosis. Nature Cell Biology 13:1108-1115. https://doi.org/10.1038/ncb2310

109. Wang L, Leite de Oliveira R, Wang C, et al (2017) High-Throughput Functional Genetic and Compound Screens Identify Targets for Senescence Induction in Cancer. Cell Rep 21:773-783. https://doi.org/10.1016/j.celrep.2017.09.085

110. Wang L-X, Wang J-D, Chen J-J, et al (2016) Aurora A Kinase Inhibitor AKI603 Induces Cellular Senescence in Chronic Myeloid Leukemia Cells Harboring T315I Mutation. Sci Rep 6:35533. https://doi.org/10.1038/srep35533

111. Zheng F-M, Long Z-J, Hou Z-J, et al (2014) A Novel Small Molecule Aurora Kinase Inhibitor Attenuates Breast Tumor-Initiating Cells and Overcomes Drug Resistance. Mol Cancer Ther 13:1991-2003. https://doi.org/10.1158/1535-7163.MCT-13-1029 
112. Liu Y, Hawkins OE, Vilgelm AE, et al (2015) Combining an Aurora Kinase Inhibitor and a Death Receptor Ligand/Agonist Antibody Triggers Apoptosis in Melanoma Cells and Prevents Tumor Growth in Preclinical Mouse Models. Clin Cancer Res 21:5338-5348. https://doi.org/10.1158/10780432.CCR-15-0293

113. Tikhmyanova N, Little JL, Golemis EA (2010) CAS proteins in normal and pathological cell growth control. Cellular and Molecular Life Sciences 67:1025-1048. https://doi.org/10.1007/s00018-0090213-1

114. Do T-V, Xiao F, Bickel LE, et al (2014) Aurora kinase A mediates epithelial ovarian cancer cell migration and adhesion. Oncogene 33:539-549. https://doi.org/10.1038/onc.2012.632

115. Mahankali M, Henkels KM, Speranza F, Gomez-Cambronero J (2015) A non-mitotic role for Aurora kinase $A$ as a direct activator of cell migration upon interaction with PLD, FAK and Src. J Cell Sci 128:516-526. https://doi.org/10.1242/jcs.157339

116. WU J, YANG L, SHAN Y, et al (2016) AURKA promotes cell migration and invasion of head and neck squamous cell carcinoma through regulation of the AURKA/Akt/FAK signaling pathway. Oncol Lett 11:1889-1894. https://doi.org/10.3892/ol.2016.4110

117. Wang L -h., Xiang J, Yan M, et al (2010) The Mitotic Kinase Aurora-A Induces Mammary Cell Migration and Breast Cancer Metastasis by Activating the Cofilin-F-actin Pathway. Cancer Research 70:9118-9128. https://doi.org/10.1158/0008-5472.CAN-10-1246

118. Kanellos G, Frame MC (2016) Cellular functions of the ADF/cofilin family at a glance. Journal of Cell Science 129:3211-3218. https://doi.org/10.1242/jcs.187849

119. D'Assoro AB, liu T, Quatraro C, et al (2014) The mitotic kinase Aurora-A promotes distant metastases by inducing epithelial-to-mesenchymal transition in ERa+ breast cancer cells. Oncogene 33:599-610. https://doi.org/10.1038/onc.2012.628

120. Wang J, Nikhil K, Viccaro K, et al (2017) Phosphorylation-dependent regulation of ALDH1A1 by Aurora kinase A: insights on their synergistic relationship in pancreatic cancer. BMC Biol 15:10. https://doi.org/10.1186/s12915-016-0335-5

121. Wang J, Nikhil K, Viccaro K, et al (2017) The Aurora-A-Twist1 axis promotes highly aggressive phenotypes in pancreatic carcinoma. J Cell Sci 130:1078-1093. https://doi.org/10.1242/jcs.196790

122. Dongre A, Weinberg RA (2019) New insights into the mechanisms of epithelial-mesenchymal transition and implications for cancer. Nat Rev Mol Cell Biol 20:69-84. https://doi.org/10.1038/s41580-018-0080-4

123. Wang F, Li H, Yan X-G, et al (2015) Alisertib induces cell cycle arrest and autophagy and suppresses epithelial-to-mesenchymal transition involving PI3K/Akt/mTOR and sirtuin 1-mediated signaling pathways in human pancreatic cancer cells. Drug Des Devel Ther 9:575-601. https://doi.org/10.2147/DDDT.S75221

124. Grant R, Abdelbaki A, Bertoldi A, et al (2018) Constitutive regulation of mitochondrial morphology by Aurora $\mathrm{A}$ kinase depends on a predicted cryptic targeting sequence at the $\mathrm{N}$-terminus. Open Biology 8:170272. https://doi.org/10.1098/rsob.170272 
125. Chang C-R, Blackstone $C$ (2010) Dynamic regulation of mitochondrial fission through modification of the dynamin-related protein Drp1: Chang \& Blackstone. Annals of the New York Academy of Sciences 1201:34-39. https://doi.org/10.1111/j.1749-6632.2010.05629.x

126. Kaestner $P$, Stolz A, Bastians $H$ (2009) Determinants for the efficiency of anticancer drugs targeting either Aurora-A or Aurora-B kinases in human colon carcinoma cells. Mol Cancer Ther 8:20462056. https://doi.org/10.1158/1535-7163.MCT-09-0323

127. Durlacher CT, Li Z-L, Chen X-W, et al (2016) An update on the pharmacokinetics and pharmacodynamics of alisertib, a selective Aurora kinase A inhibitor. Clinical and Experimental Pharmacology and Physiology 43:585-601. https://doi.org/10.1111/1440-1681.12571

128. Manfredi MG, Ecsedy JA, Chakravarty A, et al (2011) Characterization of Alisertib (MLN8237), an investigational small-molecule inhibitor of aurora A kinase using novel in vivo pharmacodynamic assays. Clin Cancer Res 17:7614-7624. https://doi.org/10.1158/1078-0432.CCR-11-1536

129. Kelly KR, Ecsedy J, Medina E, et al (2011) The novel Aurora A kinase inhibitor MLN8237 is active in resistant chronic myeloid leukaemia and significantly increases the efficacy of nilotinib. J Cell Mol Med 15:2057-2070. https://doi.org/10.1111/j.1582-4934.2010.01218.x

130. Zhou N, Singh K, Mir MC, et al (2013) The investigational Aurora kinase A inhibitor MLN8237 induces defects in cell viability and cell-cycle progression in malignant bladder cancer cells in vitro and in vivo. Clin Cancer Res 19:1717-1728. https://doi.org/10.1158/1078-0432.CCR-12-2383

131. Shimomura T, Hasako S, Nakatsuru Y, et al (2010) MK-5108, a Highly Selective Aurora-A Kinase Inhibitor, Shows Antitumor Activity Alone and in Combination with Docetaxel. Molecular Cancer Therapeutics 9:157-166. https://doi.org/10.1158/1535-7163.MCT-09-0609

132. Shan W, Akinfenwa PY, Savannah KB, et al (2012) A Small-Molecule Inhibitor Targeting the Mitotic Spindle Checkpoint Impairs the Growth of Uterine Leiomyosarcoma. Clinical Cancer Research 18:3352-3365. https://doi.org/10.1158/1078-0432.CCR-11-3058

133. Tagal V, Wei S, Zhang $W$, et al (2017) SMARCA4-inactivating mutations increase sensitivity to Aurora kinase A inhibitor VX-680 in non-small cell lung cancers. Nat Commun 8:14098. https://doi.org/10.1038/ncomms14098

134. Amin M, Minton SE, LoRusso PM, et al (2016) A phase I study of MK-5108, an oral aurora a kinase inhibitor, administered both as monotherapy and in combination with docetaxel, in patients with advanced or refractory solid tumors. Invest New Drugs 34:84-95. https://doi.org/10.1007/s10637015-0306-7

135. Fletcher GC, Brokx RD, Denny TA, et al (2011) ENMD-2076 Is an Orally Active Kinase Inhibitor with Antiangiogenic and Antiproliferative Mechanisms of Action. Molecular Cancer Therapeutics 10:126137. https://doi.org/10.1158/1535-7163.MCT-10-0574

136. Shiomitsu K, Sajo E, Rubin C, Sehgal I (2016) The radiosensitizing effect of the aurora kinase inhibitors, ENMD-2076, on canine mast cell tumours in vitro: Radiosensitizing effect of ENMD-2076. Vet Comp Oncol 14:13-27. https://doi.org/10.1111/vco.12046

137. Yee KWL, Chen H-WT, Hedley DW, et al (2016) A phase I trial of the aurora kinase inhibitor, ENMD2076 , in patients with relapsed or refractory acute myeloid leukemia or chronic myelomonocytic leukemia. Invest New Drugs 34:614-624. https://doi.org/10.1007/s10637-016-0375-2 
138. Mazumdar A, Henderson YC, El-Naggar AK, et al (2009) Aurora kinase A inhibition and paclitaxel as targeted combination therapy for head and neck squamous cell carcinoma. Head Neck 31:625634. https://doi.org/10.1002/hed.21007

139. Lin Y, Richards FM, Krippendorff B-F, et al (2012) Paclitaxel and CYC3, an aurora kinase A inhibitor, synergise in pancreatic cancer cells but not bone marrow precursor cells. Br J Cancer 107:16921701. https://doi.org/10.1038/bjc. 2012.450

140. Sehdev V, Katsha A, Ecsedy J, et al (2013) The combination of alisertib, an investigational Aurora kinase A inhibitor, and docetaxel promotes cell death and reduces tumor growth in preclinical cell models of upper gastrointestinal adenocarcinomas: Alisertib \& Docetaxel Inhibit Tumor Growth. Cancer 119:904-914. https://doi.org/10.1002/cncr.27801

141. Qi W, Cooke LS, Liu X, et al (2011) Aurora inhibitor MLN8237 in combination with docetaxel enhances apoptosis and anti-tumor activity in mantle cell lymphoma. Biochemical Pharmacology 81:881-890. https://doi.org/10.1016/j.bcp.2011.01.017

142. Caputo E, Miceli R, Motti ML, et al (2014) AurkA inhibitors enhance the effects of B-RAF and MEK inhibitors in melanoma treatment. J Transl Med 12:216. https://doi.org/10.1186/s12967-014-0216-z

143. Pathria G, Garg B, Borgdorff V, et al (2016) Overcoming MITF-conferred drug resistance through dual AURKA/MAPK targeting in human melanoma cells. Cell Death Dis 7:e2135. https://doi.org/10.1038/cddis.2015.369

144. Shah KN, Bhatt R, Rotow J, et al (2019) Aurora kinase A drives the evolution of resistance to thirdgeneration EGFR inhibitors in lung cancer. Nat Med 25:111-118. https://doi.org/10.1038/s41591018-0264-7

145. Sumi K, Tago K, Kasahara T, Funakoshi-Tago M (2011) Aurora kinase A critically contributes to the resistance to anti-cancer drug cisplatin in JAK2 V617F mutant-induced transformed cells. FEBS Lett 585:1884-1890. https://doi.org/10.1016/j.febslet.2011.04.068

146. Damodaran AP, Vaufrey L, Gavard O, Prigent C (2017) Aurora A Kinase Is a Priority Pharmaceutical Target for the Treatment of Cancers. Trends in Pharmacological Sciences 38:687-700. https://doi.org/10.1016/j.tips.2017.05.003

147. Asteriti IA, Daidone F, Colotti G, et al (2017) Identification of small molecule inhibitors of the AuroraA/TPX2 complex. Oncotarget 8:. https://doi.org/10.18632/oncotarget.16738

148. Kong Y, Bender A, Yan A (2018) Identification of Novel Aurora Kinase A (AURKA) Inhibitors via Hierarchical Ligand-Based Virtual Screening. J Chem Inf Model 58:36-47. https://doi.org/10.1021/acs.jcim.7b00300

149. Schermelleh L, Ferrand A, Huser T, et al (2019) Super-resolution microscopy demystified. Nature Cell Biology 21:72. https://doi.org/10.1038/s41556-018-0251-8

150. Wu Y, Shroff H (2018) Faster, sharper, and deeper: structured illumination microscopy for biological imaging. Nature Methods 15:1011. https://doi.org/10.1038/s41592-018-0211-z

151. Heintzmann R, Huser T (2017) Super-Resolution Structured Illumination Microscopy. https://pubs.acs.org/doi/abs/10.1021/acs.chemrev.7b00218. Accessed 25 Apr 2019 
152. Dedecker P, Mo GCH, Dertinger T, Zhang J (2012) Widely accessible method for superresolution fluorescence imaging of living systems. PNAS 109:10909-10914. https://doi.org/10.1073/pnas.1204917109

153. Dertinger T, Colyer R, lyer G, et al (2009) Fast, background-free, 3D super-resolution optical fluctuation imaging (SOFI). PNAS 106:22287-22292. https://doi.org/10.1073/pnas.0907866106

154. Mo GCH, Ross B, Hertel F, et al (2017) Genetically encoded biosensors for visualizing live-cell biochemical activity at super-resolution. Nature Methods 14:427-434. https://doi.org/10.1038/nmeth.4221

155. Pérez de Castro I, Carmena M, Prigent C, Glover DM (2017) Aurora Kinases: Classical Mitotic Roles, Non-canonical Functions and Translational Views. Frontiers Media SA

156. Piljic A, Schultz C (2008) Simultaneous Recording of Multiple Cellular Events by FRET. ACS Chem Biol 3:156-160. https://doi.org/10.1021/cb700247q

157. Grant DM, Zhang W, McGhee EJ, et al (2008) Multiplexed FRET to Image Multiple Signaling Events in Live Cells. Biophys J 95:L69-L71. https://doi.org/10.1529/biophysj.108.139204

158. Demeautis C, Sipieter F, Roul J, et al (2017) Multiplexing PKA and ERK1\&amp;2 kinases FRET biosensors in living cells using single excitation wavelength dual colour FLIM. Scientific Reports 7:41026. https://doi.org/10.1038/srep41026

159. Ross BL, Tenner B, Markwardt ML, et al (2018) Single-color, ratiometric biosensors for detecting signaling activities in live cells. eLife 7:e35458. https://doi.org/10.7554/eLife.35458 